\title{
Calibración del modelo de capacidad de rotondas del HCM2010 a condiciones locales: caso Córdoba, Argentina
}

\author{
Gotoniel Flores Castellanoํ, Violeta Depiante ${ }^{2}$ e Jorge José Galarraga ${ }^{3}$
}

\begin{abstract}
Resumen: El presente trabajo presenta la calibración de los parámetros relevantes, intervalo crítico y de seguimiento, del modelo de capacidad de rotondas propuesto en la metodología del Highway Capacity Manual (TRB, 2010) a condiciones locales de la Provincia de Córdoba - Argentina. El estudio fue efectuado en cuatro rotondas urbanas. Se realizaron filmaciones en horas de mayor tránsito, para extraer muestras representativas y calcular los parámetros de calibración del modelo. Estos son el intervalo crítico y el intervalo de seguimiento. Se empleó el método de Máxima Verosimilitud, la Regresión Lineal y la Medición Directa para estimar los valores correspondientes. Se determinó que es posible adecuar el modelo de capacidad del HCM2010 contemplando el comportamiento de los conductores locales en función de los parámetros indicados. Se concluye que, a través de una comparación con modelos calibrados en otros países, los conductores locales presentan intervalos crítico y de seguimiento menores, lo que genera una capacidad mayor en desmedro de la seguridad. Se proponen los valores de intervalos locales que contemplen la adecuación de los modelos.
\end{abstract}

Palavras-clave: rotondas, intervalo crítico, intervalo de seguimiento.

\begin{abstract}
This paper presents the calibration of the relevant parameters, critical gap and the follow-up time, of the roundabout's capacity model from Highway Capacity Manual (TRB, 2010) to local conditions of the Córdoba Province, Argentina. The field study was developed in four roundabouts. Videotaping was made during rush hour traffic to obtain a representative sample and to calculate the calibration parameters of the model. These are the Maximum Likelihood Method, Linear Regression and Direct Measurement that were chosen as proceedings to estimate their values. It was determined that it is possible to adapt the capacity model from HCM2010 contemplating local driver behavior depending on the parameters. Through comparison with calibrated models in other countries, local drivers have smaller critical and follow-up intervals, which generate a greater capacity at the expense of safety. Local intervals which reflect model's calibration are proposed.
\end{abstract}

Keywords: roundabouts, critical gap, follow up time.

\section{INTRODUCCIÓN}

Una intersección es un área compartida por dos o más caminos y su principal función es posibilitar el cambio de dirección de la ruta (Garber y Hoel, 2007). La intersección variará su complejidad en función de los números de caminos que se crucen. Por consiguiente, todas las intersecciones serán áreas donde aparecerán puntos de conflicto tanto para vehículos como para peatones. Entre las soluciones para resolver los cambios de alineamientos en intersecciones a nivel y reducir significativamente el número y la gravedad de los conflictos, se encuentran las rotondas.

La rotonda es una intersección de forma circular en la que el tránsito se desplaza hacia la izquierda (en los países donde el tránsito circula por el lado derecho) en torno a una isla central y para su correcto funcionamiento se debe cumplir que el tránsito que va a entrar en ella, debe ceder el paso al tránsito que circula dentro de la

\footnotetext{
1 Gotoniel Flores Castellano, Maestría en Ciencias de la Ingeniería con Mención en Transporte, FCEFyN, Universidad Nacional de Córdoba, Córdoba, Argentina. (gjuniorfc@gmail.com)

2 Violeta Depiante, Maestría en Ciencias de la Ingeniería con Mención en Transporte, FCEFyN, Universidad Nacional de Córdoba, Córdoba, Argentina. (vdepiante@yahoo.com)

3 Jorge José Galarraga, Maestría en Ciencias de la Ingeniería con Mención en Transporte, FCEFyN, Universidad Nacional de Córdoba Córdoba, Argentina. (jorgala@efn.uncor.edu)
}

Manuscrito recebido em 13/08/2014 e aprovado para publicação em $18 / 03 / 2015$.

Este artigo é parte de TRANSPORTES v. 23, n. 1, 2015. ISSN: 2237-1346

(online). DOI: 10.14295/transportes.v23i1.832 misma (NCHRP 672, 2010). Además de las características señaladas, dentro de su geometría debe existir cierta curvatura y condiciones deseables para inducir determinadas velocidades vehiculares. Una rotonda es entonces un anillo de flujo circulante en sentido anti-horario hacia donde convergen o divergen distintas ramas que las interconectan con el resto del conjunto.

Existen diversas metodologías para calcular la capacidad en las rotondas, fundamentadas en una larga trayectoria de investigación teórica y empírica. Entre ellas, se destaca la descrita en el capítulo 21 del Manual de Capacidad de Carreteras de los Estados Unidos de Norteamérica en su versión reciente (TRB, 2010). La eficiencia en la operación del tránsito de vehículos en una rotonda está determinada por parámetros que influyen en su capacidad. Estas variables son, por un lado, el intervalo crítico $\left(t_{c}\right)$ que es el mínimo intervalo del flujo conflictivo que necesita un vehículo para entrar a la rotonda y por otro lado, el intervalo de seguimiento $\left(\mathrm{t}_{\mathrm{f}}\right)$, que representa el tiempo entre vehículos en cola de la rama de aproximación, que ingresan en la rotonda en un mismo intervalo crítico de la corriente conflictiva o circulante.

Por consiguiente, para conocer con relativa exactitud la capacidad de la rotonda, se deben precisar los valores de $t_{c} \mathrm{y}_{\mathrm{f}}$, que a su vez dependen de las condiciones locales de circulación del lugar bajo estudio, del comportamiento de los conductores, del volumen y composición del tránsito y de la respectiva geometría. 


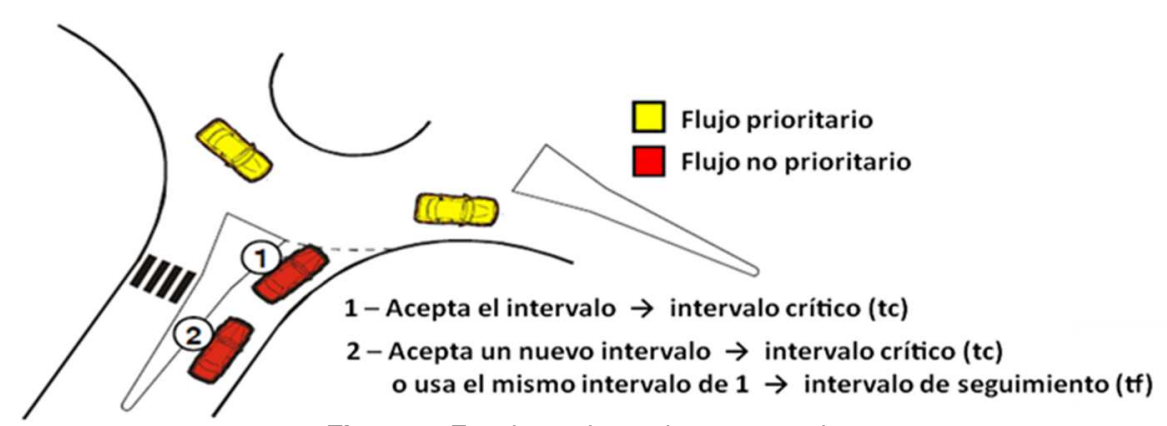

Figura 1. Funcionamiento de una rotonda

\section{REVISIÓN BIBLIOGRÁFICA}

\subsection{Funcionamiento de rotondas}

Cuando los conductores se acercan a la entrada de una rotonda deben tomar dos decisiones básicas: primero, seleccionar el carril apropiado para su destino, y segundo, "ceder el paso" a los que tienen prioridad, es decir a los que circulan por la rotonda. El proceso de toma de decisiones dentro de las rotondas es generalmente más complejo que para otros tipos de intersecciones, sobre todo porque el conductor no siempre puede ver la salida. Además, debido a que la intersección es curva, obliga a los conductores a cambiar poco a poco su dirección, lo que podría desorientar a los otros conductores sobre su destino. La decisión de "ceder el paso" produce una negociación en varios puntos de la rotonda pese a que está normalmente reglamentada la prioridad. Los conductores deben elegir un intervalo aceptable en el que puedan entrar dentro del flujo conflictivo de la rotonda.

Muchos procedimientos para hacer análisis de capacidad en rotondas se basan en modelos estocásticos. De acuerdo a esto, la capacidad depende de la disponibilidad de los intervalos y la aceptación de éstos por parte de los conductores que ingresan al tránsito de circulación. Según Raff (1950) la corriente principal (en este caso el flujo conflictivo) se puede interpretar como una oferta continua de intervalos que el conductor debe aceptar o rechazar según su criterio personal. En la Figura 1 se esquematiza el funcionamiento de una rotonda.

La teoría de aceptación de intervalos fue definida en Alemania por Harders (1968) y luego por Siegloch (1973) y Troutbeck y Brilon (1996). Los manuales de capacidad de Alemania, de Estados Unidos de Norteamérica y Suizo contemplan este criterio. Esta teoría asume que el comportamiento de los conductores es consistente y homogéneo. Las fuentes consultadas coinciden en que no es posible medir directamente los intervalos críticos, sólo se puede observar un conductor, aceptando o rechazando intervalos. Bajo un comportamiento coherente, un intervalo aceptado es una marca superior para el intervalo crítico del conductor.

Las bajas velocidades en una rotonda facilitan este proceso de aceptación (NCHRP 672, 2010). La eficiencia en la operatividad de las rotondas es mayor cuando las velocidades de circulación son menores. Este fenómeno se debe a que si el tránsito circulante es más rápido, los intervalos serán más pequeños para que el tránsito que entra los pueda aceptar cómodamente, es decir, puedan acelerar e ingresar al mismo. De esta forma habrá una menor canti- dad de intervalos aceptables con un mayor número de vehículos acumulados en la entrada, lo que se traduce en demora para entrar a la rotonda.

Cuando el volumen conflictivo se aproxima a cero, el máximo volumen de entrada está dado por 3.600 segundos por hora dividido por el intervalo de seguimiento, lo que es análogo al flujo de saturación para un movimiento que recibe verde en una intersección señalizada (NCHRP 572, 2007).

En resumen, el rendimiento operativo de rotondas depende, por un lado del comportamiento de los conductores al disponer de intervalos en la corriente conflictiva; y por otro lado, al igual que otras intersecciones, su operación está influenciada por su geometría (número de carriles, ancho de entradas, diámetro, etc.).

\subsection{Estimación de los intervalos críticos y de seguimiento}

Para estimar los parámetros de intervalo crítico $\left(\mathrm{t}_{\mathrm{c}}\right)$ e intervalo de seguimiento $\left(\mathrm{t}_{\mathrm{f}}\right)$ existen varias técnicas. Por un lado, para determinarlos de forma conjunta se tiene el análisis de regresión (entre el número de conductores que aceptan un intervalo dado versus el tamaño de dicho intervalo). Para ello debe existir cola continua en las ramas que entran en la rotonda. Por otro lado para estimarlos separadamente, el Método de Máxima Verosimilitud para encontrar el intervalo crítico ha demostrado ser muy eficiente (Troutbeck, 1992; Kyte et al., 1996; Brilon et al., 1997) у una Medición Directa permitirá obtener, mediante un promedio, los tiempos de seguimiento. Además existe la posibilidad de estimación conjunta mediante la relación entre la capacidad de la entrada (C) y del volumen conflictivo $\left(\mathrm{V}_{\mathrm{C}}\right)$ medidos en campo (Depiante y Galarraga, 2013).

El modelo de Máxima Verosimilitud utiliza los valores individuales de los intervalos medidos. Sólo se consideran el máximo intervalo rechazado y el intervalo aceptado de cada vehículo en forma individual. El intervalo crítico se encontraría entre ambos si el comportamiento del conductor fuera consistente y homogéneo por lo que, asumiendo una distribución log-normal para intervalos críticos, se pueden obtener mediante iteraciones sucesivas los parámetros de la distribución. El intervalo máximo rechazado indica el tiempo mínimo en que un conductor con un comportamiento coherente aceptaría el intervalo para ingresar a la corriente conflictiva. El intervalo crítico corresponde a la media de los intervalos críticos de los conductores y se utiliza para representar la conducta global de la población.

Para poder determinar el intervalo de seguimiento por Medición Directa, deben promediarse los intervalos 
Tabla 1. Intervalos críticos y de seguimiento propuestos por el HCM2010

\begin{tabular}{llll}
\hline Configuración & & $\boldsymbol{t}_{\boldsymbol{c}}$ & $\boldsymbol{t}_{\boldsymbol{f}}$ \\
\hline \multirow{2}{*}{ Entrada de un carril } & Un carril de circulación & 5,19 & 3,19 \\
\cline { 2 - 4 } & $\begin{array}{l}\text { Dos carriles de circulaci- } \\
\text { ón }\end{array}$ & 4,11 & 3,19 \\
\hline $\begin{array}{l}\text { Entrada de dos carriles } \\
\text { con dos carriles de circu- } \\
\text { lación }\end{array}$ & Carril izquierdo & 4,29 & 3,19 \\
\cline { 2 - 4 } & Carril derecho & 4,11 & 3,19 \\
\hline
\end{tabular}

Tabla 2. Modelos de Capacidad del HCM2010 para diferentes configuraciones

\begin{tabular}{lll}
\hline Configuración & & Modelo de capacidad \\
\hline \multirow{2}{*}{ Entrada de un carril } & Un carril de circulación & $c=1.130 \times e^{\left(-0,0010 v_{c}\right)}$ \\
\cline { 2 - 3 } & $\begin{array}{l}\text { Dos carriles de circulaci- } \\
\text { Ón }\end{array}$ & $c=1.130 \times e^{\left(-0,0007 v_{c}\right)}$ \\
\hline $\begin{array}{l}\text { Entrada de dos carriles } \\
\text { con dos carriles de circu- } \\
\text { lación }\end{array}$ & Carril izquierdo & $c_{e, L}=1.130 \times e^{\left(-0,00075 v_{c}\right)}$ \\
\cline { 2 - 3 } & Carril derecho & $c_{e, \mathrm{R}}=1.130 \times e^{\left(-0,0007 v_{c}\right)}$ \\
\hline Donde: & $\begin{array}{l}c: \text { capacidad de un carril de entrada [aphpc]; } \\
v_{c}: \text { flujo conflictivo [aphpc]; } \\
c_{e, R}: \text { capacidad del carril derecho de entrada [aphpc] } ; \mathrm{y} \\
c_{e, L} \text { : capacidad del carril izquierdo de entrada [aphpc]. }\end{array}$ \\
\hline
\end{tabular}

medidos de los vehículos que se encuentran en cola e ingresan en un mismo intervalo del flujo conflictivo o circulante de la rotonda. Es requisito indispensable para poder efectuar el cálculo, que dichos vehículos se encuentren en cola.

El método de Regresión Lineal consiste ajustar una función al conjunto de datos dados para obtener una relación empírica de predicción y proporcionar un modelo teórico que no está disponible (Canavos, 1988). Para aplicar este método deberá haber condiciones de cola continua a fin de obtener resultados satisfactorios. Se ajusta la Regresión Lineal entre los valores promedios de tamaño de intervalo (variable dependiente) y el número de vehículos que ingresan en este intervalo promedio (variable independiente). La pendiente de la recta de regresión es el valor asumido para el tiempo de seguimiento $\left(\mathrm{t}_{\mathrm{f}}\right)$, por ser el tiempo que permite el paso de un vehículo adicional (de "i" a "i+1"). El intervalo crítico se estima como la suma de la ordenada al origen más la mitad del tiempo de seguimiento, debido a que con menos de este valor no entraría ningún vehículo.

\subsection{Modelo de capacidad del HCM2010}

En el modelo del HCM2010, Ecuación 1, la capacidad se ha desarrollado como una expresión exponencial coincidiendo con el modelo de Siegloch (Kyte et al., 1996) y propuesto para rotondas (NCHRP 572, 2007) con estimaciones de los parámetros A y B sobre la base de teoría de la aceptación de intervalos en función de los intervalos crítico y de seguimiento como se muestra en las Ecuaciones 2 y 3 . El parámetro A que afecta a la exponencial depende solamente del valor del intervalo de seguimiento, en cambio el parámetro B también depende del valor del intervalo crítico.

$$
c=A e^{-B v_{c}}
$$

donde:

$$
A=\frac{3600}{t_{f}}
$$

$$
B=\frac{t_{c}-t_{f} / 2}{3600}
$$

Dicha relación permite obtener la capacidad de la entrada a partir de flujo conflictivo si se calculan los parámetros A y $\mathrm{B}$ a partir de los valores de intervalos crítico y de seguimiento.

La Tabla 1 muestra los valores de los intervalos críticos y de seguimiento para la realidad norteamericana y la Tabla 2 los modelos para estimar la capacidad de cada carril de entrada para las distintas geometrías de la rotonda para esos valores de intervalos.

Para el caso de un carril de circulación, si hay dos carriles de entrada la capacidad se duplica frente a la de un carril de entrada, ya que ambos carriles presentan la misma capacidad.

Para el caso de dos carriles de circulación las capacidades son mayores. Puede advertirse también que la capacidad de un único carril de entrada es igual a la del carril derecho de dos carriles de entrada. En este caso la capacidad, al aumentar un carril, no alcanza a duplicarse ya que la del carril izquierdo es algo inferior.

La Figura 2 muestra las curvas de capacidad de los modelos propuestos por el HCM2010.

\section{RELEVAMIENTO Y PROCESAMIENTO DE DATOS DE CAMPO}

Las rotondas relevadas están ubicadas en zonas urbanas de la ciudad y provincia de Córdoba en Argentina. Las tres primeras corresponden a rotondas con dos carriles de circulación (2c) y la cuarta con uno solo (1c). Las dos primeras presentan dos carriles de entrada (2e) y las dos restantes entradas de un solo carril (1e). En este sentido, se seleccionaron dos rotondas con dos carriles de circulación y dos carriles de entrada $(2 \mathrm{c}-2 \mathrm{e})$, una rotonda con dos carriles de circulación y un carril de entrada $(2 c-1 e)$, y por último una rotonda con un carril de circulación y un carril de entrada $(1 \mathrm{c}-1 \mathrm{e})$.

1. Rotonda "Nudo Vial Mitre" (RNM 2c-2e).

2. Rotonda "Rodríguez del Busto" (RRB 2c-2e). 


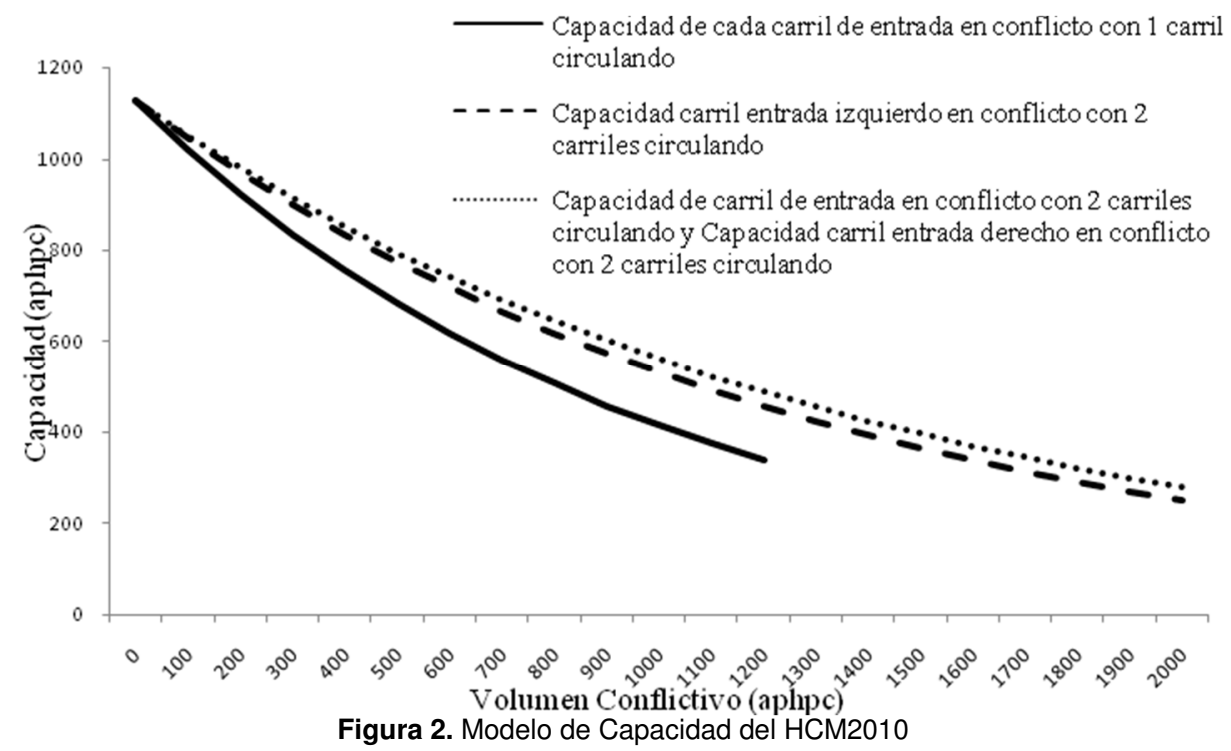

Tabla 3. Características geométricas de las rotondas

\begin{tabular}{|c|c|c|c|c|c|c|c|c|c|c|}
\hline \multirow[b]{2}{*}{ Rotonda } & \multirow[b]{2}{*}{$\begin{array}{l}\emptyset \\
\text { círculo } \\
\text { Inscrito } \\
(\boldsymbol{m})\end{array}$} & \multirow[b]{2}{*}{$\begin{array}{l}\emptyset \\
\text { Isla } \\
(\mathrm{m})\end{array}$} & \multicolumn{3}{|c|}{ Carriles de entrada } & \multicolumn{3}{|c|}{ Carriles de circulación } & \multirow[b]{2}{*}{$\begin{array}{l}\text { Pendiente } \\
(\%)\end{array}$} & \multirow[b]{2}{*}{$\begin{array}{l}\text { Ángulo de } \\
\text { entrada }\end{array}$} \\
\hline & & & $\begin{array}{l}\text { Ancho } \\
\text { Total } \\
\text { (m) }\end{array}$ & $\begin{array}{l}\text { Ancho } \\
\text { por } \\
\text { carril } \\
(m)\end{array}$ & $\begin{array}{l}\text { Radio } \\
\text { de } \\
\text { curva } \\
(m)\end{array}$ & $\begin{array}{l}\text { Ancho } \\
\text { total } \\
\text { calzada } \\
(m)\end{array}$ & $\begin{array}{l}\text { Ancho } \\
\text { total } \\
\text { carriles } \\
(\mathrm{m})\end{array}$ & $\begin{array}{l}\text { Ancho } \\
\text { por } \\
\text { carril } \\
(m)\end{array}$ & & \\
\hline RNM $2 c-2 e$ & 80 & 58 & 8,4 & 4,2 & 20,8 & 10,2 & 7 & 3,5 & 2,4 & $49^{\circ}$ \\
\hline RRB $2 c-2 e$ & 56 & 30 & 9,2 & 4,6 & 45,9 & 12 & 7,8 & 3,6 & $-1,3$ & $25^{\circ}$ \\
\hline $\operatorname{RLS} 2 \mathrm{c}-1 \mathrm{e}$ & 70 & 47 & 5,1 & -- & 12,3 & 11,4 & 7,8 & 3,9 & 0,9 & $19^{\circ}$ \\
\hline RGB $1 \mathrm{c}-1 \mathrm{e}$ & 38 & 20 & 5,4 & -- & 5,6 & 9 & 5,4 & -- & 1,3 & $68^{\circ}$ \\
\hline
\end{tabular}

3. Rotonda "Lino Spilimbergo" (RLS 2c-1e).

4. Rotonda "Avenida Goycochea - Avenida Bodereau" (RGB 1c-1e).

Se identificaron los horarios pico del tránsito en días hábiles. Se realizaron los relevamientos en horarios desde las 7:30 hasta las 9: 00 hs., desde las 12:30 hasta las 14:00 hs., y desde las 17:00 hasta las 19:00 hs. en días martes, miércoles y jueves. En cada rotonda los períodos de filmación tuvieron una duración aproximada de una hora en cada período pico diferentes, totalizando 12 horas de filmación. No existió ningún feriado próximo durante la recolección de datos que pudiera afectar las mediciones de tránsito.

Los videos en formato digital fueron revisados mediante un computador personal con la ayuda de un software de visualización de video para la extracción de datos tránsito conflictivo y volúmenes de entrada en las rotondas y tiempos de cruce de ingresos a la rotonda. La Tabla 3 resume las características geométricas de los elementos de las rotondas estudiadas.

La revisión de los videos permitió registrar y medir los intervalos entre vehículos, valores de parámetros requeridos para efectuar la calibración del modelo del HCM2010. Los tiempos requeridos se pudieron extraer con el empleo de un computador personal, de un cronómetro con tiempos parciales y un software comercial de visualización de video. Luego se procedió a conformar las bases de datos para incluir los datos registrados y proceder a efectuar los cálculos. Entre los datos registrados, se tiene el lugar, la fecha, la hora, los vehículos involucrados, tanto en la entrada como circulando, la cantidad de vehículos que entran en los intervalos del flujo conflictivo, los intervalos aceptados y los mayores intervalos rechazados. Se utilizó como referencia para el registro de tiempos una marca en el video que fue una línea de parada en el ingreso por el acceso considerado. Del mismo modo, se registraron de forma directa, los tiempos de seguimiento, los cuales fueron promediados posteriormente. Corresponde señalar que además de procesar los datos para cada una de las rotondas, se combinaron los datos de las rotondas de igual configuración de carriles. Para esto, se unieron los datos de la rotonda RNM $2 c-2 e$ y RRB $2 c-2 e$ a fin de realizar un análisis y comparación de los resultados.

\subsection{Estimación del intervalo crítico mediante el método de Máxima Verosimilitud}

Este método fue utilizado para determinar el intervalo crítico de forma independiente del tiempo de seguimiento. Los tiempos medidos en las filmaciones fueron el "intervalo aceptado" por cada vehículo y el "mayor intervalo rechazado" por el mismo vehículo. Se entiende que el intervalo aceptado es el intervalo que utiliza un vehículo en la entrada de la rotonda para ingresar entre dos vehículos consecutivos del flujo conflictivo que circula dentro de la misma. El mayor intervalo rechazado representa el mayor tiempo entre dos vehículos consecutivos del flujo conflictivo, que dispuso el vehículo que espera para ingresar y no fue utilizado.

Cuando hay una cola de vehículos esperando para ingresar a la rotonda y se da el caso de que algunos de estos autos ingresan, entonces el primer auto sería el único que registra un valor de "mayor intervalo rechazado", ya que el resto de los vehículos que entraron al flujo conflictivo con él, tienen un valor de "mayor intervalo rechaza- 
Tabla 4. Características geométricas de las rotondas

\begin{tabular}{|c|c|c|c|c|c|c|c|c|}
\hline \multirow[b]{2}{*}{ Carril } & \multicolumn{2}{|c|}{ RNM $2 c-2 e$} & \multicolumn{2}{|l|}{$R R B 2 c-2 e$} & \multicolumn{2}{|c|}{$\begin{array}{l}\text { Combinación } \\
2 c-2 e\end{array}$} & \multirow{2}{*}{$\begin{array}{l}R L S \\
2 c-1 e \\
\text { Único }\end{array}$} & \multirow{2}{*}{$\begin{array}{l}\text { RGB } \\
1 c-1 e\end{array}$} \\
\hline & Izquierdo & Derecho & Izquierdo & Derecho & Izquierdo & Derecho & & \\
\hline $\mathrm{n}$ & 408 & 297 & 286 & 103 & 694 & 400 & 166 & 198 \\
\hline $\mathrm{t}_{\mathrm{c}}(\mathrm{s})$ & 3,34 & 3,34 & 3,43 & 3,17 & 3,34 & 3,34 & 3,56 & 3,60 \\
\hline $\operatorname{var}\left(t_{c}\right)(s)$ & 0,07 & 0,08 & 0,10 & 0,01 & 0,07 & 0,08 & 0,17 & 0,08 \\
\hline$\sigma\left(\mathrm{t}_{\mathrm{c}}\right)(\mathrm{s})$ & 0,26 & 0,29 & 0,31 & 0,12 & 0,26 & 0,29 & 0,41 & 0,28 \\
\hline Int. Confianza & $95 \%$ & $95 \%$ & $95 \%$ & $95 \%$ & $95 \%$ & $95 \%$ & $95 \%$ & $95 \%$ \\
\hline $\mathrm{t}_{\mathrm{c}}$ superior $(\mathrm{s})$ & 3,37 & 3,38 & 3,47 & 3,19 & 3,36 & 3,37 & 3,62 & 3,64 \\
\hline $\mathrm{t}_{\mathrm{c}}$ inferior $(\mathrm{s})$ & 3,32 & 3,31 & 3,39 & 3,15 & 3,32 & 3,32 & 3,50 & 3,56 \\
\hline
\end{tabular}

Tabla 5. Resumen de intervalos de seguimiento para cada rotonda y carril obtenido por promedios de intervalos

\begin{tabular}{|c|c|c|c|c|c|c|c|c|}
\hline \multirow[b]{2}{*}{ Carril } & \multicolumn{2}{|c|}{$R N M 2 c-2 e$} & \multicolumn{2}{|c|}{$R R B 2 c-2 e$} & \multicolumn{2}{|c|}{$\begin{array}{l}\text { Combinación } \\
2 c-2 e\end{array}$} & \multirow{2}{*}{$\begin{array}{l}R L S \\
2 c-1 e \\
U_{n i c o}\end{array}$} & \multirow{2}{*}{$\begin{array}{l}R G B \\
1 c-1 e \\
\text { Único }\end{array}$} \\
\hline & Izquierdo & Derecho & Izquierdo & Derecho & Izquierdo & Derecho & & \\
\hline $\mathrm{n}$ & 1021 & 1204 & 511 & 167 & 1532 & 1371 & 203 & 197 \\
\hline $\operatorname{tf}(s)$ & 2,11 & 2,05 & 2,20 & 2,14 & 2,14 & 2,06 & 2,31 & 2,34 \\
\hline $\operatorname{var}\left(\mathrm{t}_{\mathrm{f}}\right)(\mathrm{s})$ & 0,16 & 0,15 & 0,20 & 0,16 & 0,17 & 0,15 & 0,16 & 0,12 \\
\hline$\sigma\left(\mathrm{t}_{\mathrm{f}}\right)(\mathrm{s})$ & 0,40 & 0,39 & 0,44 & 0,40 & 0,41 & 0,39 & 0,40 & 0,34 \\
\hline Int. Confianza & $95 \%$ & $95 \%$ & $95 \%$ & $95 \%$ & $95 \%$ & $95 \%$ & $95 \%$ & $95 \%$ \\
\hline $\mathrm{t}_{\mathrm{f}}$ superior $(\mathrm{s})$ & 2,13 & 2,07 & 2,24 & 2,20 & 2,16 & 2,08 & 2,37 & 2,39 \\
\hline$t_{\mathrm{f}}$ inferior $(s)$ & 2,09 & 2,03 & 2,17 & 2,08 & 2,12 & 2,04 & 2,26 & 2,29 \\
\hline
\end{tabular}

do" igual a cero, por no haber tenido la posibilidad de rechazar ninguno.

En la Tabla 4 se resumen los resultados obtenidos, indicando el carril correspondiente, el tamaño de la muestra (n), el $\mathrm{t}_{\mathrm{c}}$ medio, la varianza (var), la desviación estándar $(\sigma)$ junto con los límites superior e inferior con un intervalo de confianza del $95 \%$ para el $\mathrm{t}_{\mathrm{c}}$.

Como se advierte, la cantidad de datos (n) de la Rotonda RNM 2c-2e fue muy superior a la rotonda RRB 2c$2 \mathrm{e}$, esto se refleja en los intervalos críticos obtenidos en la combinación de ambas.

Los valores de intervalos críticos resultaron superiores en el caso de rotondas con acceso de entrada de un solo carril respecto de las de dos carriles. Cuando existen dos carriles los conductores tienden a aprovechar más los intervalos disponibles, especialmente el carril de la derecha que tiene más facilidad de ingreso que el de la izquierda.

\subsection{Estimación del intervalo de seguimiento mediante Medición Directa por promedio de intervalos}

El método de Medición Directa por promedio de intervalos fue utilizado para determinar el intervalo de seguimiento de manera independiente del intervalo crítico.
Se procedió a calcular los límites superiores e inferiores para cada una de las rotondas, con un intervalo de confianza del 95\%. En la Tabla 5 se resumen los resultados, indicando el carril correspondiente, el tamaño de la muestra (n), el t t promedio, la varianza (var), la desviación estándar $(\sigma)$ y los límites superior e inferior.

\subsection{Estimación conjunta de intervalo crítico y de seguimiento mediante Regresión Lineal}

El método de Regresión Lineal permite estimar de manera conjunta el intervalo crítico e intervalo de seguimiento en condiciones de cola. Para esto se determinaron cantidades de vehículos (n) que ingresan en la rotonda en cada intervalo de duración " $t$ " en el flujo conflictivo $\left(V_{C}\right)$. Los intervalos crítico y de seguimiento fueron calculados a partir de las regresiones efectuadas con la totalidad de los datos y no con los promedios. Esto es debido a que se tienen suficientes datos y los R2 son elevados. Las Figuras 3 y 4 muestran el ajuste por regresión y la ecuación para el carril izquierdo y el carril derecho para el caso combinando ambas rotondas " $2 \mathrm{c}-2 \mathrm{e}$ ".

En la Tabla 6 se resumen los resultados de la Regresión Lineal, por cada carril, el tamaño de la muestra para cada "n", el total de casos con el tiempo promedio, el

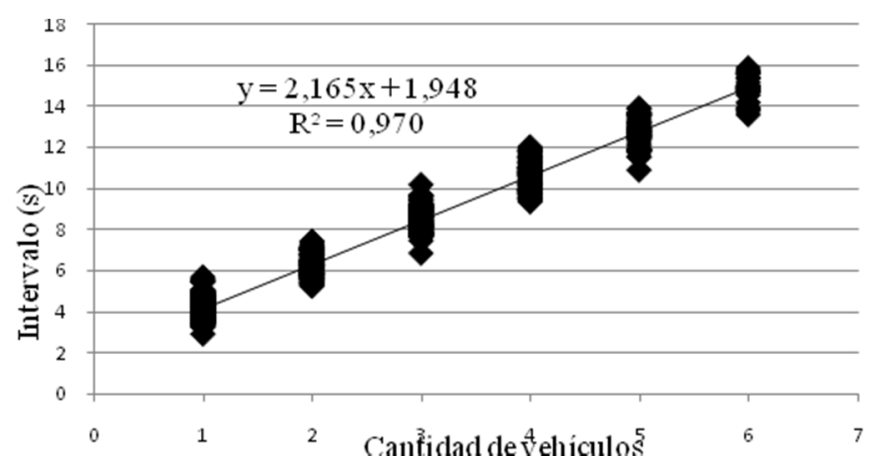

Figura 3. Curva de Regresión "Combinación 2c - 2e", Carril Izquierdo 


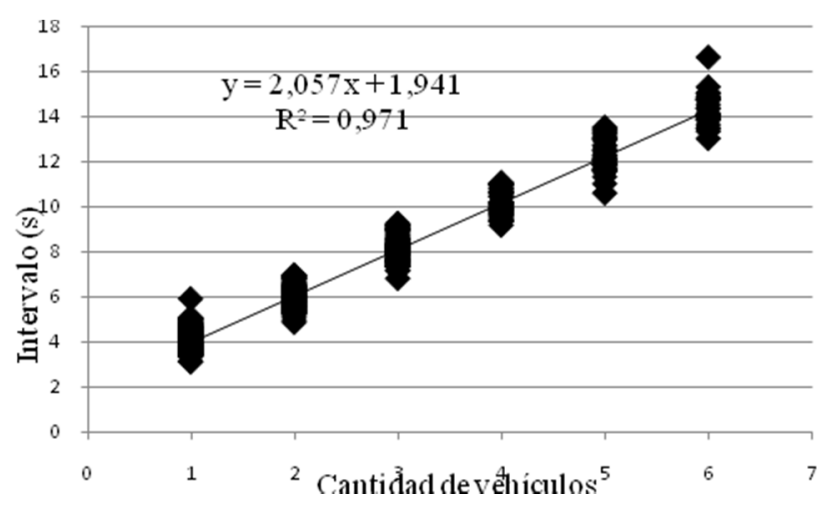

Figura 4. Curva de Regresión "Combinación 2c-2e", Carril Derecho

Tabla 6. Resumen de intervalos críticos y de seguimiento obtenido por Regresión Lineal

\begin{tabular}{|c|c|c|c|c|c|c|c|c|c|c|c|c|c|c|c|c|}
\hline \multirow{3}{*}{$\frac{\text { Carril }}{\mathrm{n}}$} & \multicolumn{4}{|c|}{$R N M 2 c-2 e$} & \multicolumn{4}{|c|}{$R R B 2 c-2 e$} & \multicolumn{4}{|c|}{$\begin{array}{l}\text { Combinación } \\
2 c-2 e\end{array}$} & \multirow{2}{*}{\multicolumn{2}{|c|}{$\begin{array}{l}R L S \\
2 c-1 e \\
\text { Único } \\
\end{array}$}} & \multirow{2}{*}{\multicolumn{2}{|c|}{$\begin{array}{l}R G B \\
1 c-1 e \\
\text { Único } \\
\end{array}$}} \\
\hline & \multicolumn{2}{|c|}{ Izquierdo } & \multicolumn{2}{|c|}{ Derecho } & \multicolumn{2}{|c|}{ Izquierdo } & \multicolumn{2}{|c|}{ Derecho } & \multicolumn{2}{|c|}{ Izquierdo } & \multicolumn{2}{|c|}{ Derecho } & & & & \\
\hline & casos & tprom. & casos & $\mathrm{t}_{\text {prom. }}$ & casos & tprom. & casos & $\mathrm{t}_{\text {prom. }}$ & casos & $\mathrm{t}_{\text {prom. }}$ & casos & $t_{\text {prom. }}$ & casos & $t_{\text {prom. }}$ & casos & $t_{\text {prom. }}$ \\
\hline 1 & 239 & 4,11 & 151 & 4,05 & 115 & 4,15 & 52 & 4,02 & 354 & 4,12 & 203 & 4,05 & 39 & 4,19 & 39 & 4,22 \\
\hline 2 & 155 & 6,20 & 123 & 5,98 & 62 & 6,30 & 12 & 5,94 & 217 & 6,23 & 135 & 5,97 & 20 & 6,44 & 20 & 6,54 \\
\hline 3 & 90 & 8,47 & 98 & 8,10 & 31 & 8,61 & 08 & 8,03 & 121 & 8,51 & 106 & 8,09 & 10 & 8,53 & 05 & 9,02 \\
\hline 4 & 62 & 10,53 & 44 & 10,19 & 25 & 10,97 & & & 87 & 10,66 & 46 & 10,20 & & & & \\
\hline 5 & 37 & 12,65 & 40 & 12,27 & 16 & 12,92 & & & 53 & 12,73 & 40 & 12,27 & & & & \\
\hline 6 & 19 & 14,88 & 26 & 14,33 & & & & & 26 & 14,91 & 26 & 14,33 & & & & \\
\hline Total & 602 & & 482 & & 249 & & 72 & & 858 & & 556 & & 69 & & 64 & \\
\hline $\mathrm{R}^{2}$ & 0,972 & & 0,971 & & 0,960 & & 0,90 & & 0,970 & & 0,971 & & 0,861 & & 0,9 & \\
\hline $\mathrm{m}$ & 2,148 & & 2,058 & & 2,227 & & 1,98 & & 2,165 & & 2,057 & & 2,061 & & 2,364 & \\
\hline b & 1,950 & & 1,940 & & 1,910 & & 2,030 & & 1,948 & & 1,941 & & 2,148 & & 1,846 & \\
\hline $\mathrm{t}_{\mathrm{c}}(\mathrm{s})$ & 3,02 & & 2,97 & & 3,02 & & 3,02 & & 3,03 & & 2,97 & & 3,18 & & 3,03 & \\
\hline $\mathrm{t}_{\mathrm{f}}(\mathrm{s})$ & 2,15 & & 2,06 & & 2,23 & & 1,99 & & 2,17 & & 2,06 & & 2,06 & & 2,36 & \\
\hline
\end{tabular}

coeficiente de determinación (R2), la pendiente (m), el corte en la ordenada (b) y los valores para $t_{c} \mathrm{y}_{\mathrm{f}}$ resultantes de la regresión.

Para mayor detalle sobre el relevamiento y procesamiento de la información puede consultarse Flores Castellano (2013).

\section{COMPARACIÓN DE LOS INTERVALOS LOCALES, HCM2010 Y OTRAS REALIDADES}

Como antecedente de esta investigación, se revisó un trabajo desarrollado en la ciudad de Montevideo, Uruguay (Caimi, 2008), donde se estimaron los valores de $t_{c} y$ $\mathrm{t}_{\mathrm{f}}$ en cuatro rotondas. Debe señalarse que las rotondas analizadas en Montevideo, son todas de configuración 2c2e, y se estimó el $t_{c} \mathrm{y}_{\mathrm{f}}$ sin discriminar los carriles de entrada. De igual manera se realizaron comparaciones con estudios de Italia (Gazzarri et al., 2012), de California, USA (Xu y Tian, 2007), y con datos de otras fuentes, incluyendo el reporte NCHRP 572, con información de seis estados de los EE.UU. (Washington, Maryland, Maine, Michigan, Oregón y Vermont) y de Alemania.

Tabla 7. Comparación de los valores de $t_{c}, t_{f}, " A$ " y "B" del carril izquierdo en rotondas $(2 c-2 e)$ de Córdoba y otros países

\begin{tabular}{lllll}
\hline Rotondas Multicarriles & $\mathrm{t}_{\mathrm{c}}$ & $\mathrm{t}_{\mathrm{f}}$ & $\mathrm{A}$ & $\mathrm{B}$ \\
\hline RNM 2c - 2e & 3,34 & 2,11 & 1706 & 0,0006 \\
RRB 2c - 2e & 3,43 & 2,20 & 1636 & 0,0006 \\
NCHRP 572 & 4,50 & 3,40 & 1059 & 0,0008 \\
California & 4,70 & 2,20 & 1636 & 0,0010 \\
Uruguay & 3,40 & 2,20 & 1636 & 0,0006 \\
HCM2010 & 4,29 & 3,19 & 1130 & 0,00075 \\
Alemania & 4,40 & 3,20 & 1125 & 0,00078 \\
Italia & 3,85 & 2,59 & 1390 & 0,00071 \\
\hline
\end{tabular}

Tabla 8. Comparación de los valores de $t_{c}, t_{f}$ " "A" y "B" del carril derecho en rotondas $(2 c-2 e)$ de Córdoba y otros países

\begin{tabular}{lllll}
\hline Rotondas Multicarriles & $\mathrm{t}_{\mathrm{c}}$ & $\mathrm{t}_{\mathrm{f}}$ & $\mathrm{A}$ & $\mathrm{B}$ \\
\hline RNM 2c - 2e & 3,34 & 2,05 & 1756 & 0,0006 \\
RRB 2c - 2e & 3,17 & 2,14 & 1682 & 0,0006 \\
NCHRP 572 & 4,20 & 3,10 & 1161 & 0,0007 \\
California & 4,40 & 2,20 & 1636 & 0,0009 \\
Uruguay & 3,40 & 2,20 & 1636 & 0,0006 \\
HCM2010 & 4,11 & 3,19 & 1129 & 0,00070 \\
Alemania & 4,40 & 3,20 & 1125 & 0,00078 \\
Italia & 3,64 & 2,63 & 1369 & 0,00065 \\
\hline
\end{tabular}


Tabla 9. Comparación de los valores de $\mathrm{t}_{\mathrm{c}}, \mathrm{t}_{\mathrm{f}}$, "A" $\mathrm{y}$ "B" en rotondas $(2 \mathrm{c}-1 \mathrm{e})$ de Córdoba y del HCM2010

\begin{tabular}{lllll}
\hline Rotondas & $\boldsymbol{t}_{\boldsymbol{c}}$ & $\boldsymbol{t}_{\boldsymbol{f}}$ & $\boldsymbol{A}$ & $\boldsymbol{B}$ \\
\hline RLS 2c - 1e & 3,56 & 2,31 & 1556 & 0,0007 \\
HCM2010 & 4,11 & 3,19 & 1130 & 0,0007 \\
\hline
\end{tabular}

Tabla 10. Comparación de los valores de $t_{c}, t_{f}$, "A" y "B" en rotondas $(1 c-1 e)$ de Córdoba y otros países

\begin{tabular}{lllll}
\hline Rotondas & $\boldsymbol{t}_{\boldsymbol{c}}$ & $\boldsymbol{t}_{\boldsymbol{f}}$ & $\boldsymbol{A}$ & $\boldsymbol{B}$ \\
\hline RGB 1c - 1e & 3,60 & 2,34 & 1538 & 0,0007 \\
NCHRP 572 & 5,10 & 3,20 & 1125 & 0,0010 \\
California & 4,80 & 2,50 & 1440 & 0,0010 \\
HCM2010 & 5,19 & 3,19 & 1130 & 0,0010 \\
Alemania & 4,40 & 3,20 & 1125 & 0,00078 \\
Italia & 3,83 & 2,64 & 1364 & 0,00070 \\
\hline
\end{tabular}

Tabla 11. Intervalos críticos y de seguimiento recomendados para condiciones locales

\begin{tabular}{llll}
\hline Configuración & & $\boldsymbol{t}_{\boldsymbol{c}}$ & $\boldsymbol{t}_{\boldsymbol{f}}$ \\
\hline \multirow{2}{*}{ Entrada de un carril } & Un carril de circulación & 3,60 & 2,34 \\
\cline { 2 - 4 } & $\begin{array}{l}\text { Dos carriles de circulaci- } \\
\text { Ón }\end{array}$ & 3,60 & 2,34 \\
\hline $\begin{array}{l}\text { Entrada de dos carriles } \\
\text { con dos carriles de circu- } \\
\text { lación }\end{array}$ & Carril izquierdo & 3,34 & 2,11 \\
\cline { 2 - 4 } & Carril derecho & 3,34 & 2,05 \\
\hline
\end{tabular}

Los Tablas 7 a 10 reportan los valores comparativos de intervalos críticos y de seguimiento para rotondas $2 \mathrm{c}-$ 2e carril izquierdo y derecho, rotondas $2 \mathrm{c}-1 \mathrm{e}$ y rotondas 1c-1e, y también los valores locales de los parámetros A y B utilizados en la formulación de capacidad del HCM2010. Corresponde consignar que para las rotondas locales se reportan valores obtenidos por el método de Máxima Verosimilitud (para $\mathrm{t}_{\mathrm{c}}$ ) y por Medición Directa (para $\left.t_{f}\right)$.

Se observa que los valores de $t_{c} y t_{f}$ locales son menores que los valores referenciales de otros países, lo que significa un mayor valor estimado para la capacidad de la entrada. Se advierte que los valores y las curvas de capacidad resultantes son similares a las rotondas de Uruguay. La curva de las rotondas en California comienza muy aproximada a las anteriores, por tener $\mathrm{t}_{\mathrm{f}}$ muy parecidos, sin embargo la capacidad decrece más rápido con el aumento del volumen conflictivo por tener un $t_{c}$ mayor. En comparación con los valores aportados por el NCHRP 572, los de Alemania y del HCM2010, siempre las rotondas en Córdoba y Uruguay mantienen valores de capacidad muy por encima de estas. Similarmente, para el carril de la derecha se mantiene el mismo comportamiento. Los valores de $t_{c}$ para Italia resultan intermedios entre los de USA - Alemania y Argentina - Uruguay.

En el caso de las rotondas $2 \mathrm{c}-1 \mathrm{e}$, el $\mathrm{t}_{\mathrm{c}} \mathrm{y}$ el $\mathrm{t}_{\mathrm{f}}$ es significativamente más bajo que el referido por el HCM2010. Las curvas de capacidad para Córdoba muestran valores mayores que el resto, advirtiéndose que la curva de California posee una capacidad similar para valores de $\mathrm{V}_{\mathrm{C}}$ bajos. Esto se debe a que en California los $t_{f}$ medidos son muy similares a los locales, sin embargo por tener un $t_{c}$ mayor, la curva de capacidad decae con mayor pendiente. Para las rotondas 1c - 1e, los valores capacidad para condiciones locales están muy por encima del resto excepto en el caso de Italia donde los valores se aproximan más a nuestra realidad reflejando un comportamiento de los conductores más semejante.

\section{CONCLUSIONES Y RECOMENDACIONES}

La calibración del modelo de capacidad del HCM2010 depende de la determinación de los $t_{\mathrm{c}} \mathrm{y}$ los $\mathrm{t}_{\mathrm{f}}$ para cada una de las configuraciones de las rotondas. Teniendo en cuenta los valores locales, se confirma la presencia de intervalos menores que indican un comportamiento más agresivo del conductor y la generación de capacidades más elevadas en las rotondas locales. En general el comportamiento de los conductores en Argentina es más agresivo en relación a los conductores de otros países. Otros estudios locales reflejan este hecho, como por ejemplo los referidos a tiempos de verde efectivo en intersecciones semaforizadas (Albrieu y Galarraga, 2012). También puede influir que el diseño geométrico (parámetros) de las rotondas en algunos casos privilegian los movimientos de acceso.

Comparando los valores obtenidos por el método de Regresión Lineal con respecto a los estimados por Máxima Verosimilitud y Medición Directa, se advierte que se obtienen valores menores de $\mathrm{t}_{\mathrm{c}} \mathrm{y}_{\mathrm{f}}$, lo que aumenta la capacidad en relación a los otros dos métodos. Esto podría deberse a que el cálculo por Regresión Lineal requiere de la existencia de cola continua. Normalmente cuando eso ocurre los vehículos que ingresan a la rotonda tienden a optimizar el uso de los intervalos disponibles.

En vista de la comparación de los métodos, con resultados más conservadores, se procede a la calibración del modelo de capacidad del HCM2010 con los parámetros de $t_{c} \mathrm{y}_{\mathrm{f}}$ estimados de forma independientes (Máxima Verosimilitud y promedios de la Medición Directa), que con un nivel de confiabilidad del $95 \%$, incluye en la mayoría de los casos los valores obtenidos mediante los otros procedimientos.

Para el caso dos carriles de circulación y dos carriles de entrada, se recomiendan los valores estimados en la RNM $2 c-2 e$, ya que en ésta se registró una cantidad muy importante de datos, con cola continua y resultados robustos, que al combinarse con los datos de la rotonda RRB 2c 
Tabla 12. Modelos de capacidad recomendados para condiciones locales

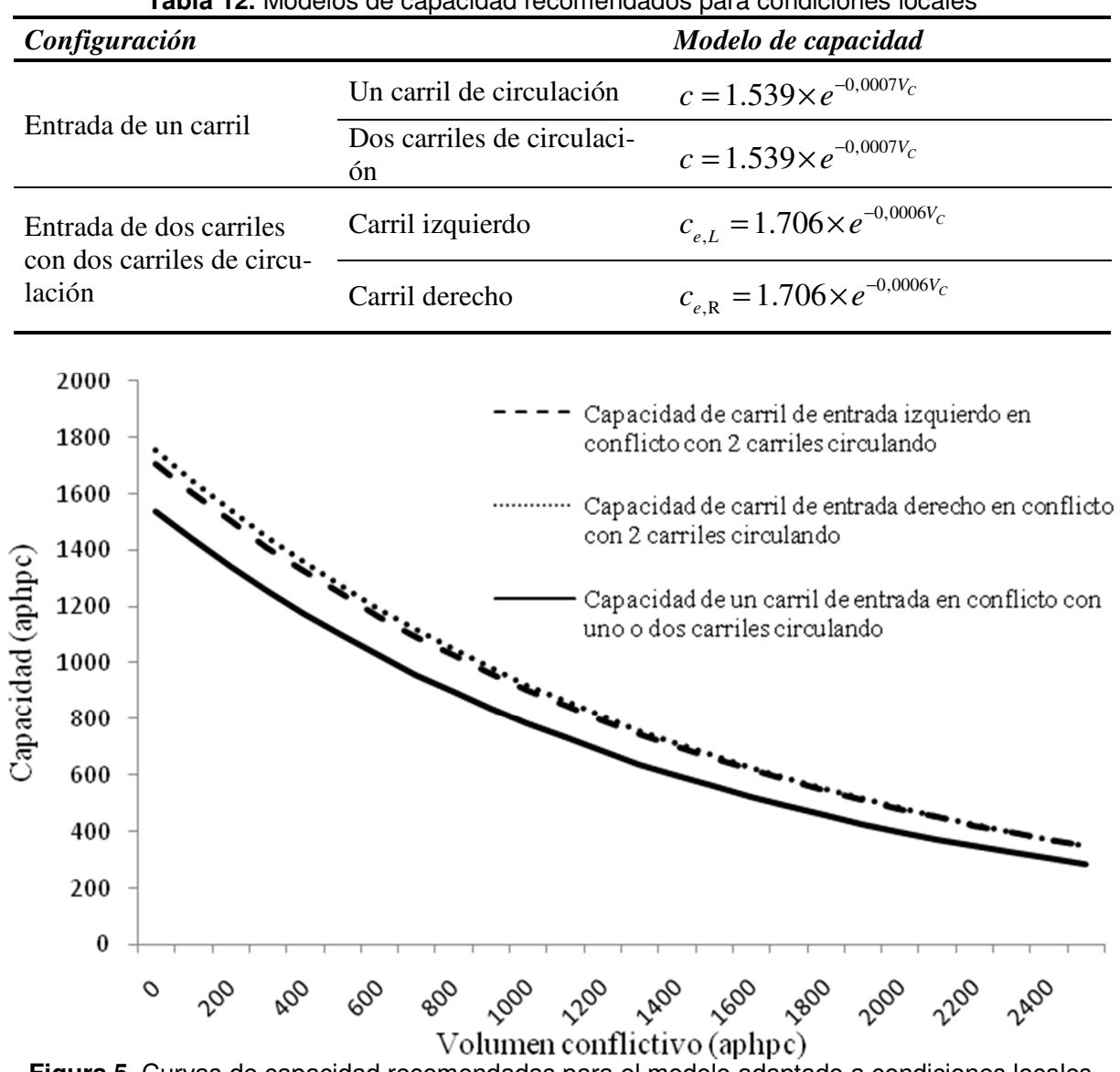

Figura 5. Curvas de capacidad recomendadas para el modelo adaptado a condiciones locales

$-2 \mathrm{e}$, arrojaron resultados que reflejaron las mismas características de la primera.

Para el caso de uno o dos carriles de circulación y un carril de entrada, se recomiendan los valores estimados para la RGB 1c - 1e, que además de ser muy parecidos a los de la rotonda RLS $2 \mathrm{c}-1 \mathrm{e}$, son los más conservadores. En la Tabla 11 se presentan los $t_{c} \mathrm{y}_{\mathrm{f}}$ recomendados para la calibración del Modelo del HCM2010.

A partir del $t_{c} y t_{f}$ señalados, se obtienen los valores de los coeficientes "A" y "B" del modelo de capacidad, Ecuaciones 2 y 3, los cuales se muestran en la Tabla 12.

En la Figura 5 se muestran las curvas de capacidad que representan el modelo para las rotondas de Córdoba, con uno y dos carriles de entrada.

Se advierte que los resultados locales difieren de los propuestos por el HCM2010. Es de destacar que el intervalo de seguimiento, que permanece invariable en el HCM2010 para todos los casos, muestra diferencias entre las entradas de uno o dos carriles en condiciones locales. Además, en condiciones locales, las rotondas con uno o dos carriles de circulación para entradas de un carril presentan parámetros similares.

Este es un trabajo inicial para conocer las condiciones de operación de rotondas urbanas del Área Metropolitana de Córdoba y puede servir de precedente para otras evaluaciones de tránsito en este tipo de intersecciones y soluciones futuras. La composición del tránsito es casi en su totalidad de vehículos livianos (autos y camionetas), con mínimos porcentajes de participación de los otros modos.

\section{REFERENCIAS}

ALBRIEU, M. y GALARRAGA, J. (2012) Recomendaciones para la aplicación de la metodología del HCM para intersecciones semaforizadas en Argentina. XVI Congreso Argentino de Vialidad y Tránsito, Córdoba, Argentina.

BRILON, W., KÖNIG, R. y TROUTBECK, R. (1997) Useful Estimation Procedures for Critical Gaps. Proceeding of Third Int. Symposium on Intersections without Traffic Signals, Portland, Oregon, U.S.A. DOI: 10.1016/S0965-8564(98)00048-2

CAIMI, M. (2008) Rotondas: Estimación del Intervalo Crítico y del Tiempo de Seguimiento y su Aplicación en las Medidas de Eficiencia, Disertación Maestría en Ciencias de la Ingeniería Mención en Transporte, Facultad de Ciencias Exactas, Físicas y Naturales, Universidad Nacional de Córdoba, Argentina.

CANAVOS, G. (1988) Probabilidad y Estadística, Aplicaciones y Métodos, Editorial McGraw Hill, México, DF.

DEPIANTE, V. y GALARRAGA, J. (2013) Minor Street Gaps and Capacity at Unsignalized Intersections in Argentina. XIII WCTR, Rio de Janeiro, Brasil.

FLORES CASTELLANO, G. J. (2013) Capacidad de Rotondas: Calibración del Modelo del HCM 2010 a Condiciones Locales. Disertación Maestría en Ciencias de la Ingeniería Mención en Transporte. Facultad de Ciencias Exactas, Físicas y Naturales, Universidad Nacional de Córdoba, Argentina.

GARBER, N. y HOEL, L. (2007) Ingeniería de Tránsito y Carreteras, $3^{\text {ra }}$ edición, Cengage Learning, México, D.F. 
GAZZARRI A., MARTELLO M. T., PRATELLI1 A. AND SOULEYRETTE R. R. (2012) Estimation of gap acceptance parameters for HCM 2010 roundabout capacity model applications. WIT Transactions on The Built Environment, Vol 128, WIT Press, ISSN 1743-3509 (on-line) DOI: 10.2495/UT120271

HARDERS, J. (1968) Die Leistungs faehigkeitnicht signal geregelter staedtischer Verkehrsknoten (Capacity of unsignalized urban intersections). Strassenbau und Strassenverkehrstechnik, Vol. 76. Bundesministerfuer Verkehr, Abt. Strassenbau, Bonn.

KYTE, M., TIAN, Z., MIR, Z., HAMEEDMANSOOR, Z., KITTELSON, W., VANDEHEY, M., ROBINSON, B., BRILON, W., BONDZIO, L., WU, N. y TROUTBECK, R. (1996) Capacity and Level of Service at Unsignalized Intersections. Final Report: Volume 1. National Cooperative Highway Research Program 3-46.

RAFF, M. y HART, J. (1950) A volume warrant for urban stop sign. The Eno Foundation for Highway Traffic Control.

SIEGLOCH, W. (1973) Capacity calculations for unsignalized intersections. Schriftenreihe Strassenbau, Vol. 154.

NCHRP Report 572 (2007) Roundabouts in the United States, National Cooperative Highway Research Program, Transportation Research Board of the National Academies, Washington D.C., USA.

NCHRP Report 672 (2010) Roundabouts: A Informational Guide, Second Edition, National Cooperative Highway Research Program, Transportation Research Board of the National Academies, Washington D.C., USA.

TRB (2010) Highway Capacity Manual. Chapter 21: Roundabouts, Volumen 3: Interrupted Flow, Transportation Research Board of the National Academies, Washington D.C., USA.

TROUTBECK, R. (1992) Estimating the critical acceptance gap from traffic movements, Queensland University of Technology, Australia.

TROUTBECK, R. y BRILON, W. (1996) Unsignalized Intersection Theory. Chapter 8, página de internet: http://www.fhwa.dot.gov/publications/research/operations/tft/cha p8.pdf

XU, F. y TIAN, Z. (2007) Roundabout Geometric Design Guidance. Report $\mathrm{N}^{\circ}$ F/CA/RI-2006/13 California Department of Transportation, Department of Civil Engineering, California State University, U.S.A. 